\title{
Zero-One Law for Connectivity in Superposition of Random Key Graphs on Random Geometric Graphs
}

\author{
Y. Tang and Q. L. Li \\ College of Mathematics and Computer Science, Hunan Normal University, Changsha 410081, China \\ Correspondence should be addressed to Q. L. Li; liqiaoliang@eyou.com
}

Received 24 June 2015; Revised 11 October 2015; Accepted 18 October 2015

Academic Editor: Filippo Cacace

Copyright (C) 2015 Y. Tang and Q. L. Li. This is an open access article distributed under the Creative Commons Attribution License, which permits unrestricted use, distribution, and reproduction in any medium, provided the original work is properly cited.

\begin{abstract}
We study connectivity property in the superposition of random key graph on random geometric graph. For this class of random graphs, we establish a new version of a conjectured zero-one law for graph connectivity as the number of nodes becomes unboundedly large. The results reported here strengthen recent work by the Krishnan et al.
\end{abstract}

\section{Introduction}

Random key graph (RKG), also known as uniform random intersection graph, is a random graph defined below. Consider a set with $n$ nodes and another key pool with $P_{n}$ keys; we assume each node randomly chooses $K_{n}$ distinct keys for its key ring; two nodes can establish a secure link between them if they share at least one common key in their key rings.

The random key graph is naturally associated with the random key predistribution scheme of Eschenauer and Gligor [1] for wireless sensor networks (WSNs). A WSN is a collection of distributed sensor devices that are able to communicated wirelessly and supports wide range of applications such as health and environment monitoring, imaging, tracking, and biomedical research; see [2]. These applications require all nodes in the network to be within communication range and to be connected with high probability.

Some partial results concerning the connectivity of RKGs were given in [3-5]. In [6], Rybarczyk gave asymptotic tight bounds for the thresholds of the connectivity, phase transition, and diameter of the largest connected component in RKGs for all ranges of $K_{n}$.

With the advent of ad hoc sensor networks, an interesting class of random graphs, namely, random geometric graphs (RGGs), has gained new importance and its properties have been the subject of much study. Here the nodes are randomly distributed in a finite Euclidean space and there is an edge between two nodes if the Euclidean distance between them is below a specified threshold. Much work has been done on graph theoretic properties of such graph, comprehensively summarized in the monograph of [7].

Recently, there is interest in random graphs in which an edge is determined by more than one random property, that is, superposition of different random graphs. The superposition of ER random graphs over RGGs has been of interest for quite some time now. Recent work on such random graphs is in $[8,9]$ where connectivity properties and the distribution of isolated nodes are analyzed. And the superposition of ER random graphs on RKGs is considered in [10]. Such a graph is constructed as follows: a RKG is first formed based on the key distribution and each edge in this graph is deleted with a specified probability.

The superposition of RKGs on RGGs is first studied in [11]. The $n$ nodes are distributed in a finite Euclidean space and each node is assigned a key ring of $K_{n}$ distinct keys drawn randomly from a pool of $P_{n}$ keys. Two nodes have an edge if and only if they share at least one common key in their key rings and their Euclidean distance is at most $r_{n}$. Pietro et al. [11] have shown that under the scaling $\pi r_{n}^{2} K_{n}^{2} / P_{n}=c(\log n / n)$, the one-law that this class of random graphs is connected follows if $r_{n}>0$ and $c>20 \pi$. Another notable work is due by Krzywdziński and Rybarczyk [12], where the authors have improved these results and established the one-law for $c>8$ without any constraint on $r_{n}$. Recently, Krishnan et al. [13] 
have shown that for large $n$, this class of random graphs will be connected if $K_{n} \geq 2, r_{n}$ and $P_{n}$ are selected such that

$$
\begin{aligned}
K_{n}, P_{n} & \longrightarrow \infty, \\
\frac{K_{n}^{2}}{P_{n}} & \longrightarrow 0, \\
P_{n} & \geq 2 K_{n}, \\
P_{n} & \geq \sigma n r_{n}^{2}, \\
\pi r_{n}^{2} \frac{K_{n}^{2}}{P_{n}} & >\frac{2 \pi}{1-\gamma} \frac{\log n}{n},
\end{aligned}
$$

for any $\sigma>0$ and $0<\gamma<1$. They also observed that for large $n$ and $0<c_{1}<\infty$, the probability that this class of random graphs is disconnected is at least $e^{-c_{1}} / 4$ if the scaling satisfies

$$
\pi r_{n}^{2} \frac{K_{n}^{2}}{P_{n}}=\frac{\log n+c_{1}}{n} .
$$

The connectivity in the superposition of RKGs on RGGs is still studied in this paper. Assuming that $P_{n} \geq n$, we show that given $n \pi r_{n}^{2} K_{n}^{2} / P_{n}=\log n+c_{n}$, this class of random graphs is disconnected if $c_{n} \rightarrow-\infty$, and for $c_{n} \rightarrow \infty$, this class of random graphs is connected.

In this paper, we use standard, asymptotic notations: $a_{n}=$ $\Theta\left(b_{n}\right), a_{n}=\omega\left(b_{n}\right), a_{n}=o\left(b_{n}\right)$, and $a_{n} \sim b_{n}$ for $\exists_{c, C>0} c b_{n} \leq$ $a_{n} \leq C b_{n}, a_{n} / b_{n} \rightarrow \infty, a_{n} / b_{n} \rightarrow 0$, and $a_{n} / b_{n} \rightarrow 1$, respectively, all limits are taken as $n \rightarrow \infty$. The phrase "with high probability" (abbreviated whp) means with probability tending to one as $n$ tends to infinity.

The rest of the paper is organized as follows. Our main result is presented in Section 2. Namely, the theorem concerning zero-one law for graph connectivity is presented. Section 3 contains technical proof of Theorem 1. Finally, Section 4 discusses prospects of establishing tighter connectivity thresholds in the superposition of RKGs on RGGs.

\section{Main Result}

The $n$ nodes are uniformly and independently distributed in $\mathfrak{R}=[0,1]^{2}$. Let $x_{i} \in \mathfrak{R}$ be the location of point $i$. A key pool with $P_{n}$ cryptographic keys is designated for the network of $n$ nodes. Node $i$ randomly chooses a subset $S_{i}$ of keys from the key pool with $\left|S_{i}\right|=K_{n}$. Our interest is in the random graph $G\left(P_{n}, K_{n}, r_{n}\right)$ with $n$ nodes and edges formed as follows. An edge $(i, j), 1 \leq i<j \leq n$, is present in $G\left(P_{n}, K_{n}, r_{n}\right)$ if both of the following two conditions are satisfied:

$$
\begin{gathered}
E_{1}:\left\|x_{i}-x_{j}\right\| \leq r_{n}, \\
E_{2}: S_{i} \cap S_{j} \neq \emptyset,
\end{gathered}
$$

where $\|\cdot\|$ represents the Euclidean norm. Condition $E_{1}$ produces a random geometric graph with the transmission range $r_{n}$. Imposing condition $E_{2}$ on $E_{1}$ retains the edges of the random geometric graph for which the two nodes share at least one common key. Thus $G\left(P_{n}, K_{n}, r_{n}\right)$ is a superposition of RKG on RGG.

In the following, to avoid technicalities which obscure the main ideas, we will neglect edge effects resulting due to the fact that $n$ nodes are distributed uniformly and independently over a folded unit square $\mathfrak{R}=[0,1]^{2}$ with continuous boundary conditions and a node is close to the boundary of $\mathfrak{R}$. Throughout the paper, we set $n \pi r_{n}^{2}=d_{n}$, where $d_{n}=$ $\omega(\log n)$ and $d_{n}=o\left(n^{(1-\delta) / 4}\right)$ for any small $0<\delta<1$. The following theorem gives zero-one law for the connectivity of a superposition of RKG on RGG.

Theorem 1. Let $K_{n} \geq 2, K_{n}, P_{n} \rightarrow \infty, K_{n}^{2} / P_{n} \rightarrow 0, P_{n} \geq n$. Then

(i) if $\pi r_{n}^{2} K_{n}^{2} / P_{n}=\left(\log n+c_{n}\right) / n$ and $c_{n} \rightarrow-\infty$, then with high probability $G\left(P_{n}, K_{n}, r_{n}\right)$ is disconnected;

(ii) if $\pi r_{n}^{2} K_{n}^{2} / P_{n}=\left(2 \log n+c_{n}\right) / n$ and $c_{n} \rightarrow \infty$, then with high probability $G\left(P_{n}, K_{n}, r_{n}\right)$ is connected.

The first part of the Theorem 1 is proved by using the second moment method, that is, considering the probability of finding at least one isolated node in the network for a random graph $G\left(P_{n}, K_{n}, r_{n}\right)$. The second part takes a slightly different approach, we assume that $\mathfrak{R}=[0,1]^{2}$ is entirely coved by circle cells of radius $r_{n} / 2$. Connectivity of $G\left(P_{n}, K_{n}, r_{n}\right)$ is ensured as follows: (1) every circle cell is dense; namely, every circle cell has $\Theta\left(n r_{n}^{2}\right)$ nodes inside it; (2) the overlapping structure of any two adjacent circle cells has at least one common node; and (3) the nodes in any circle cell form a connected subgraph.

\section{Proof of Theorem 1}

Before proceeding, we first introduce some definitions and auxiliary lemmas. For $i=1,2, \ldots, n$, let $X_{i}=1$ if node $i$ is isolated in $G\left(P_{n}, K_{n}, r_{n}\right)$ and $X=\sum_{i=1}^{n} X_{i}$. Then, $X$ is exactly the number of isolated nodes in $G\left(P_{n}, K_{n}, r_{n}\right)$. Let $|S|$ denote the cardinality of a set $S$ and let $E(X), \operatorname{Var}(X)$ denote the expectation and variance of random variable $X$, respectively. As a special case of Markov's inequality the first moment method states that

$$
\operatorname{Pr}(X \geq 1) \leq E X,
$$

and the second moment method (special case of Tschebyscheff's inequality) states that

$$
\operatorname{Pr}(X=0) \leq \frac{\operatorname{Var} X}{(E X)^{2}} .
$$

If $X$ is a binomial distributed random variable, $\lambda=E X$ and for any $t \geq 0$, then we will use the following variants of Chernoff's inequality (see [14]):

$$
\begin{aligned}
& \operatorname{Pr}(X \geq \lambda+t) \leq \exp \left(-\frac{t^{2}}{2(\lambda+t / 3)}\right), \\
& \operatorname{Pr}(X \leq \lambda-t) \leq \exp \left(-\frac{t^{2}}{2 \lambda}\right)
\end{aligned}
$$


Of course, it is easy to check that

$$
\beta_{n}=1-\frac{\left(\begin{array}{c}
P_{n}-K_{n} \\
K_{n}
\end{array}\right)}{\left(\begin{array}{c}
P_{n} \\
K_{n}
\end{array}\right)} \sim \frac{K_{n}^{2}}{P_{n}}
$$

under the assumption that $\lim _{n \rightarrow \infty}\left(K_{n}^{2} / P_{n}\right)=0$, where $\beta_{n}$ is the probability that two nodes share at least one common key. Throughout, we make use of the standard bounds

$$
\left(\begin{array}{l}
n \\
u
\end{array}\right) \leq\left(\frac{e n}{u}\right)^{u}
$$

valid for all $u, n=1,2, \ldots$ with $u \leq n$. Finally, we note the equation

$$
(1-p)^{m} \sim e^{-m p}
$$

holds if $m p^{2} \rightarrow 0$.

Proof of Statement (i) of Theorem 1. Let $\alpha_{n}=\pi r_{n}^{2}, \beta_{n}=$ $1-\left(\begin{array}{c}P_{n}-K_{n} \\ K_{n}\end{array}\right) /\left(\begin{array}{c}P_{n} \\ K_{n}\end{array}\right)$, where $\beta_{n}$ is the probability that two nodes share at least one common key in their key rings. By linearity of expectation, $E X=E\left(\sum_{i=1}^{n} X_{i}\right)=n E X_{i}$; hence

$$
\begin{aligned}
E X & =n E X_{i}=n\left(1-\alpha_{n} \beta_{n}\right)^{n-1} \\
& \sim n \exp \left(-(n-1) \alpha_{n} \beta_{n}\right) \\
& =n \exp \left(-(n-1) \frac{\log n+c_{n}}{n}\right) \\
& =n \exp \left(-\log n-c_{n}\right) \exp \left(\frac{\log n+c_{n}}{n}\right) \\
& \sim n \exp (-\log n) \exp \left(-c_{n}\right)=\exp \left(-c_{n}\right) \rightarrow \infty,
\end{aligned}
$$

as $c_{n} \rightarrow-\infty$. The second moment method now implies the result we require, provided that we can show that $\operatorname{Var} X=$ $o\left((E X)^{2}\right)$. Now

$$
\operatorname{Var} X=E\left(X^{2}\right)-(E X)^{2} \geq 0
$$

and so it suffices to show that $E\left(X^{2}\right)=(1+o(1))(E X)^{2}$. Note that

$$
E\left(X^{2}\right)=E X+n(n-1) E\left(X_{1} X_{2}\right)
$$

where 1, 2 are fixed nodes. Since $E X \rightarrow \infty$, it therefore suffices to prove that

$$
\frac{n(n-1) E\left(X_{1} X_{2}\right)}{(E X)^{2}} \longrightarrow 1 \text { as } n \rightarrow \infty \text {. }
$$

Note that $X_{1} X_{2}$ take the value 1 exactly when node 1 and node 2 are both isolated. Consider two discs of radius $r_{n}$ centered at $x_{1}$ and $x_{2}$; let $d=\left\|x_{1}-x_{2}\right\| ;$ the cross term $n(n-1) E\left(X_{1} X_{2}\right)$ is shown to be given by

$$
\begin{aligned}
& n(n-1) E\left(X_{1} X_{2}\right)=n(n-1) \operatorname{Pr}\left(X_{1} X_{2}=1\right) \\
& \quad=n(n-1) \operatorname{Pr}\left(X_{1} X_{2}=1 \mid d>2 r_{n}\right) \operatorname{Pr}\left(d>2 r_{n}\right) \\
& \quad+n(n-1) \operatorname{Pr}\left(X_{1} X_{2}=1 \mid r_{n}<d \leq 2 r_{n}\right) \\
& \quad \cdot \operatorname{Pr}\left(r_{n}<d \leq 2 r_{n}\right)+n(n-1) \\
& \quad \cdot \operatorname{Pr}\left(X_{1} X_{2}=1 \mid 0 \leq d \leq r_{n}\right) \operatorname{Pr}\left(0 \leq d \leq r_{n}\right) .
\end{aligned}
$$

Conditional on the range of $d$, we consider the following three cases. In each case, the conditional joint probability of two nodes being isolated can be obtained from [13].

(1) $d>2 r_{n}$ : this case happens with the probability $1-$ $4 \alpha_{n}$, and

$$
\operatorname{Pr}\left(X_{1} X_{2}=1 \mid d>2 r_{n}\right)=\left(1-2 \alpha_{n} \beta_{n}\right)^{n-2} .
$$

(2) $r_{n}<d \leq 2 r_{n}$ : this case happens with the probability $3 \alpha_{n}$, and

$$
\begin{aligned}
& \operatorname{Pr}\left(X_{1} X_{2}=1 \mid r_{n}<d \leq 2 r_{n}\right) \\
& \quad \leq\left(K_{n}+1\right) \exp \left(-(n-2)\left(2-\left\|\frac{\gamma_{n}}{\beta_{n}}-2\right\|\right) \alpha_{n} \beta_{n}\right),
\end{aligned}
$$

where $\gamma_{n}=1-\left(\begin{array}{c}P_{n}-2 K_{n} \\ K_{n}\end{array}\right) /\left(\begin{array}{c}P_{n} \\ K_{n}\end{array}\right)$. and

(3) $0 \leq d \leq r_{n}$ : this case happens with the probability $\alpha_{n}$,

$$
\begin{aligned}
& \operatorname{Pr}\left(X_{1} X_{2}=1 \mid 0 \leq d \leq r_{n}\right) \\
& \quad \leq \exp \left(-(n-2)\left(2-\left\|\frac{\gamma_{n}}{\beta_{n}}-2\right\|\right) \alpha_{n} \beta_{n}\right) .
\end{aligned}
$$

The upper bound on $n(n-1) E\left(X_{1} X_{2}\right)$ that nodes 1 and 2 are isolated if $r_{n}<d \leq 2 r_{n}$ and $0 \leq d \leq r_{n}$ is obtained together using (16) and (17). So $3 n(n-1) \alpha_{n} \operatorname{Pr}\left(X_{1} X_{2}=1\right.$ । $\left.r_{n} \leq d \leq 2 r_{n}\right)+n(n-1) \alpha_{n} \operatorname{Pr}\left(X_{1} X_{2}=1 \mid 0 \leq d \leq r_{n}\right)$ is upper-bounded as follows:

$$
\begin{aligned}
& 3 n(n-1) \alpha_{n} \operatorname{Pr}\left(X_{1} X_{2}=1 \mid r_{n} \leq d \leq 2 r_{n}\right)+n(n-1) \\
& \cdot \alpha_{n} \operatorname{Pr}\left(X_{1} X_{2}=1 \mid 0 \leq d \leq r_{n}\right) \\
& \leq \frac{n(n-1)\left(K_{n}+1\right) \alpha_{n}}{n^{2}} \exp \left(\operatorname { l o g } n \left[\left\|\frac{\gamma_{n}}{\beta_{n}}-2\right\|\right.\right. \\
& -\frac{c_{n}\left(2-\left\|\gamma_{n} / \beta_{n}-2\right\|\right)}{\log n} \\
& \left.\left.+\frac{\left(4-2\left(\left\|\gamma_{n} / \beta_{n}-2\right\|\right)\right) \alpha_{n} \beta_{n}}{\log n}\right]\right) \leq \frac{1}{\sqrt{n}} .
\end{aligned}
$$

See [13] for details.

From (14), (15), and (18), the term $n(n-1) E\left(X_{1} X_{2}\right)$ is bounded as

$$
\begin{aligned}
& n(n-1)\left(1-4 \alpha_{n}\right)\left(1-2 \alpha_{n} \beta_{n}\right)^{n-2} \\
& \quad \leq n(n-1) E\left(X_{1} X_{2}\right) .
\end{aligned}
$$


The term $n(n-1) E\left(X_{1} X_{2}\right)$ also satisfies

$$
\begin{aligned}
& n(n-1) E\left(X_{1} X_{2}\right) \\
& \quad \leq n(n-1)\left(1-4 \alpha_{n}\right)\left(1-2 \alpha_{n} \beta_{n}\right)^{n-2}+\frac{1}{\sqrt{n}} .
\end{aligned}
$$

Since $E X=n\left(1-\alpha_{n} \beta_{n}\right)^{n-1}$, if $n \rightarrow \infty$, we find that

$$
\begin{gathered}
\frac{n(n-1)\left(1-4 \alpha_{n}\right)\left(1-2 \alpha_{n} \beta_{n}\right)^{n-2}}{(E X)^{2}} \longrightarrow 1 \\
\frac{n(n-1)\left(1-4 \alpha_{n}\right)\left(1-2 \alpha_{n} \beta_{n}\right)^{n-2}+1 / \sqrt{n}}{(E X)^{2}} \longrightarrow 1 .
\end{gathered}
$$

The above two convergence formulas are true since $\alpha_{n}=$ $d_{n} / n$, where $d_{n}=\omega(\log n), d_{n}=o\left(n^{(1-\delta) / 4}\right)$, and $\alpha_{n} \beta_{n}=$ $\left(\log n+c_{n}\right) / n$. We see that (13) holds as required, which implies that

$$
\operatorname{Pr}(X=0) \leq \frac{\operatorname{Var} X}{(E X)^{2}} \longrightarrow 0
$$

as $n \rightarrow \infty$, which concludes the proof.

If $n \alpha_{n} \beta_{n}=\log n+c_{n}$ for any $c_{n} \rightarrow \infty$, we have $E X \rightarrow 0$. Then using the first moment method, we see that the probability $\operatorname{Pr}(X \geq 1) \leq E X \rightarrow 0$ holds; this implies a.a.s. there are no isolated nodes in random graph $G\left(P_{n}, K_{n}, r_{n}\right)$. The upcoming corollary is immediate from the proof of statement (i) of Theorem 1.

Corollary 2. In the model $G\left(P_{n}, K_{n}, r_{n}\right)$, let $K_{n} \geq 2, K_{n}, P_{n} \rightarrow$ $\infty, K_{n}^{2} / P_{n} \rightarrow 0, P_{n} \geq n$, and

$$
\pi r_{n}^{2} \frac{K_{n}^{2}}{P_{n}}=\frac{\log n+c_{n}}{n}
$$

(i) If $c_{n} \rightarrow-\infty$ as $n \rightarrow \infty$, then whp $G\left(P_{n}, K_{n}, r_{n}\right)$ contains at least an isolated node.

(ii) If $c_{n} \rightarrow \infty$ as $n \rightarrow \infty$, then whp $G\left(P_{n}, K_{n}, r_{n}\right)$ does not contain an isolated node.

Proof of Statement (ii) of Theorem 1 . We consider the unitarea square on $\Re=[0,1]^{2} ; \Re$ is divided into square cells of size $s_{n} \times s_{n}$, where $1 / s_{n}$ is an integer. Let the center of square cell be the center of the circle cell and let the diagonal line of the square cell be the diameter of the circle cell. In this way, $\mathfrak{R}$ is entirely covered by the circle cells. Also we let $r_{n}=\sqrt{2} s_{n}$; this means that two nodes in the same circle cell are within communicating range of each other.

Recall that $n \alpha_{n}=d_{n}$, where $\alpha_{n}=\pi r_{n}^{2}, d_{n}=\omega(\log n)$ and $d_{n}=o\left(n^{(1 / 4)(1-\delta)}\right)$. In order to complete the proof, we show the following two lemmas.

Lemma 3. (i) Every circle cell is dense; specifically, whp every circle cell has $\Theta\left(n r_{n}^{2}\right)$ nodes in it. (ii) The overlapping structure of any two adjacent circle cells whp has $\Theta\left(n s_{n}^{2}\right)$ nodes.

Proof. (i) First, we analyze the denseness of every circle cell; let $N_{i}$ denote the number of nodes in circle cell $i, 1 \leq i \leq 1 / s_{n}^{2}$. Obviously, $N_{i}$ is a binomial random variable with parameters $\left(n,(\pi / 2) s_{n}^{2}\right)$. Let $W_{i}$ denote the event that the circle cell $i$ is not dense, in other words, for any $0<\delta_{1}<1 / 2, \delta_{1} \leq \delta$, $\left|N_{i}-(\pi / 2) n s_{n}^{2}\right| \geq \delta_{1}(\pi / 2) n s_{n}^{2}$. Using Chernoff's inequalities on $N_{i}$, we have

$$
\begin{aligned}
& \operatorname{Pr}\left(N_{i} \leq\left(1-\delta_{1}\right) \frac{\pi}{2} n s_{n}^{2}\right) \leq \exp \left(-\frac{\pi}{4} n s_{n}^{2} \delta_{1}^{2}\right), \\
& \operatorname{Pr}\left(N_{i} \geq\left(1+\delta_{1}\right) \frac{\pi}{2} n s_{n}^{2}\right) \leq \exp \left(-\frac{\pi}{8} n s_{n}^{2} \delta_{1}^{2}\right) .
\end{aligned}
$$

Consequently

$$
\begin{aligned}
\operatorname{Pr}\left(W_{i}=1\right) & \leq 2 \exp \left(-\frac{\pi n s_{n}^{2} \delta_{1}^{2}}{8}\right) \\
& =2 \exp \left(-\frac{d_{n} \delta_{1}^{2}}{16}\right), \\
\operatorname{Pr}\left(\bigcup_{i=1}^{1 / s_{n}^{2}} W_{i}=1\right) & \leq \frac{1}{s_{n}^{2}} \operatorname{Pr}\left(W_{i}=1\right) \leq \exp \left(-\frac{d_{n} \delta_{1}^{2}}{32}\right) \\
& \longrightarrow 0,
\end{aligned}
$$

which implies that every circle cell is dense.

(ii) Now we consider the nodes in the overlapping structure of any two adjacent circle cells. Let $N^{\prime}$ denote the number of nodes in the overlapping structure of any two adjacent circle cells. Clearly $N^{\prime}$ is a binomial random variable with parameters $\left(n,((\pi-2) / 4) s_{n}^{2}\right)$. For any $0<\delta_{2}<1$, we also use Chernoff's inequalities on $N^{\prime}$ :

$$
\begin{aligned}
& \operatorname{Pr}\left(N^{\prime} \leq\left(1-\delta_{2}\right) \frac{\pi-2}{4} n s_{n}^{2}\right) \leq \exp \left(-\frac{\pi-2}{8} n s_{n}^{2} \delta_{2}^{2}\right), \\
& \operatorname{Pr}\left(N^{\prime} \geq\left(1+\delta_{2}\right) \frac{\pi-2}{4} n s_{n}^{2}\right) \leq \exp \left(-\frac{\pi-2}{16} n s_{n}^{2} \delta_{2}^{2}\right) .
\end{aligned}
$$

From the two above inequalities we may easily get

$$
\begin{aligned}
& \operatorname{Pr}\left(\left|N^{\prime}-\frac{\pi-2}{4} n s_{n}^{2}\right| \geq \delta_{2} \frac{\pi-2}{4} n s_{n}^{2}\right) \\
& \quad \leq 2 \exp \left(-\frac{\pi-2}{16} n s_{n}^{2} \delta_{2}^{2}\right)=2 \exp \left(-\frac{\pi-2}{32 \pi} d_{n} \delta_{2}^{2}\right)
\end{aligned}
$$$$
\longrightarrow 0 \text {. }
$$

The above expression implies that $N^{\prime}$ is very likely close to its expectation $((\pi-2) / 4) n s_{n}^{2}$. So we get our result that whp the overlapping structure of any two adjacent circle cells has at least one common node.

Lemma 4. The nodes in any circle cell form a connected subgraph; that is, for any fixed $1 \leq i \leq 1 / s_{n}^{2}$ circle cell $i$ contains 
no components of size $l, 1 \leq l \leq N_{i} / 2$, where $N_{i}$ is the number of nodes in circle cell $i$.

Proof. This part takes a slightly different approach; we consider the subgraph formed by the nodes in circle cell $i$; denote this subgraph by $G_{i}$. We will show that for any circle cell $i$ there are no components of size $1,2, \ldots, N_{i} / 2$ in $G_{i}$.

Consider any fixed circle cell $i, 1 \leq i \leq 1 / s_{n}^{2}$, for any nonempty subset $L$ of nodes in circle cell $i$; that is, $L \subseteq$ $\left\{1,2, \ldots, N_{i}\right\}$, with $1 \leq|L| \leq N_{i} / 2$; define the following events.

$C_{i}(L)$ : a subgraph induced by nodes in $L$ is connected.

$B_{i}(L): L$ is isolated in circle cell $i$; that is, there are no edges between the nodes in $L$ and the nodes in the complement $L^{C}=\left\{1,2, \ldots, N_{i}\right\}-L$. Consider

$$
\begin{aligned}
A_{i}(L) & =B_{i}(L) \cap C_{i}(L), \\
D_{i} & =\bigcup_{l=1}^{\left\lceil N_{i} / 2\right\rceil} \bigcup_{L:|L|=l} A_{i}(L) .
\end{aligned}
$$

Further, let $C_{i, l}$ and $A_{i, l}$ denote $C_{i}(L)$ and $A_{i}(L)$ with $|L|=l$, respectively. Then the sufficient condition for every circle cell a.a.s. containing no components of size $l\left(1 \leq l \leq N_{i} / 2\right)$ is to have $\operatorname{Pr}\left(D_{i}\right) / s_{n}^{2} \rightarrow 0$. Conditioned on $W_{i}$, we have

$$
\begin{aligned}
\operatorname{Pr}\left(D_{i}\right) & =\sum_{j \in\{0,1\}} \operatorname{Pr}\left(D_{i} \mid W_{i}=j\right) \operatorname{Pr}\left(W_{i}=j\right) \\
& \leq \operatorname{Pr}\left(D_{i} \mid W_{i}=0\right)+\operatorname{Pr}\left(W_{i}=1\right) .
\end{aligned}
$$

The above inequality uses bounds on the factorial, $\operatorname{Pr}\left(W_{i}=\right.$ $0) \leq 1$ and $\operatorname{Pr}\left(D_{i} \mid W_{i}=1\right) \leq 1$; from Lemma 3, we get $\operatorname{Pr}\left(W_{i}=1\right) / s_{n}^{2} \rightarrow 0$; thus we focus on showing that $\operatorname{Pr}\left(D_{i} \mid\right.$ $\left.W_{i}=0\right) / s_{n}^{2} \rightarrow 0$. Note that

$$
\begin{aligned}
\operatorname{Pr}\left(D_{i} \mid W_{i}=0\right) & =\operatorname{Pr}\left(\bigcup_{l=1}^{\left\lceil N_{i} / 2\right\rceil} \bigcup_{L:|L|=l} A_{i, l}\right) \\
& \leq \sum_{l=1}^{\left\lceil N_{i} / 2\right\rceil}\left(\begin{array}{c}
N_{i} \\
l
\end{array}\right) \operatorname{Pr}\left(A_{i, l}\right) .
\end{aligned}
$$

We let $U_{i, l}$ denote the number of distinct keys in the component of size $l$ in $G_{i}$. Adapting [5], for any $x=$ $K_{n}, \ldots, \min \left\{l K_{n}, P_{n}\right\}$, we have

$$
\begin{aligned}
\operatorname{Pr}\left(A_{i, l}\right) \leq & \operatorname{Pr}\left(U_{i, l} \leq x\right) \exp \left(-\left(N_{i}-l\right) \frac{K_{n}^{2}}{P_{n}}\right) \\
& +\operatorname{Pr}\left(C_{i, l}\right) \exp \left(-\left(N_{i}-l\right) \frac{K_{n}(x+1)}{P_{n}}\right) .
\end{aligned}
$$

From [5], we know that

$$
\begin{aligned}
\operatorname{Pr}\left(U_{i, l} \leq x\right) & \leq\left(\begin{array}{c}
P_{n} \\
x
\end{array}\right)\left(\frac{x}{P_{n}}\right)^{l K_{n}} \\
\operatorname{Pr}\left(C_{i, l}\right) & \leq l^{l-2} \beta_{n}^{l-1} .
\end{aligned}
$$

First we use the standard Poissonization technique $[7,15]$ to show the probability of having isolated nodes in any of the circle cells. Denote the circle $i$ by $A_{i}$; let $I_{i}=1$ be the event that node $i$ is isolated in $A_{i}$, and let $D_{r_{n}}\left(\widehat{v}_{i}\right)$ be the intersection of $A_{i}$ and the disk centered at position $\widehat{v}_{i} \in A_{i}$ with radius $r_{n}$, where node $i$ is at position $\widehat{v}_{i}$. Similar to the discussion in [16], the number of nodes within $D_{r_{n}}\left(\widehat{v}_{i}\right)$ follows a Poisson distribution with mean $n D_{r_{n}}\left(\widehat{v}_{i}\right)$; and to have an edge with $i$, a node not only has to be within a $D_{r_{n}}\left(\widehat{v}_{i}\right)$ but also has to share at least a key with node $i$, so the number of nodes neighboring to $i$ follows a Poisson distribution with mean $n \beta_{n} D_{r_{n}}\left(\widehat{v}_{i}\right)$. Integrating $\widehat{v}_{i}$ over $A_{i}$, the probability that node $i$ is isolated in $A_{i}$ is given by

$$
\operatorname{Pr}\left(I_{i}=1\right)=\int_{A_{i}} e^{-n \beta_{n}\left|D_{r_{n}}\left(\widehat{v}_{i}\right)\right|} d \widehat{v}_{i}
$$

The probability that there are no isolated nodes in any of the circle cells is bounded below:

$$
\begin{aligned}
\frac{1}{s_{n}^{2}} N_{i} \operatorname{Pr}\left(I_{i}=1\right) & \leq \frac{1}{s_{n}^{2}} \frac{1+\delta_{1}}{2} n \pi s_{n}^{2} \int_{A_{i}} e^{-n \beta_{n}\left|D_{r_{n}}\left(\widehat{v}_{i}\right)\right|} d \widehat{v}_{i} \\
& \leq \frac{1+\delta_{1}}{2} n \pi \exp \left(-n \beta_{n} \frac{\pi}{2} s_{n}^{2}\right)\left|A_{i}\right| \\
& \leq \frac{1+\delta_{1}}{2} n \pi \exp \left(-\frac{2 \log n+c_{n}}{4}\right) \frac{\pi}{2} s_{n}^{2} \\
& \leq \frac{1+\delta_{1}}{8} \pi d_{n} \exp \left(-\frac{2 \log n+c_{n}}{4}\right) \\
& \leq \frac{1+\delta_{1}}{8} \pi \exp \left(-\frac{1}{2} \log n+\log d_{n}\right)
\end{aligned}
$$

The second step and the third step are obtained since $\left|D_{r_{n}}\left(\widehat{v}_{i}\right)\right|=(\pi / 2) s_{n}^{2}$ and $\left|A_{i}\right|=(\pi / 2) s_{n}^{2}$, respectively. Since $d_{n}=o\left(n^{(1 / 4)(1-\delta)}\right)$, we get

$$
\frac{1}{s_{n}^{2}} N_{i} \operatorname{Pr}\left(I_{i}=1\right) \longrightarrow 0 .
$$

Next we prove that every circle cell $i$ contains no component of size $l, 2 \leq l \leq N_{i} / 2$. The sum term in (30) is evaluated in following three cases based on the size of the component $L$.

Case $1(2 \leq l \leq R)$. In the case, fewer than $(1-\epsilon) l K_{n}$ keys are assigned to the component $L$, where $0<\delta_{1} /\left(1-\delta_{1}\right)<\epsilon<1$, and $R$ is an integer. Note that

$$
\begin{aligned}
\operatorname{Pr}\left(A_{i, l}\right) \leq & \operatorname{Pr}\left(U_{i, l} \leq x\right) \exp \left(-\left(N_{i}-l\right) \frac{K_{n}^{2}}{P_{n}}\right) \\
& +\operatorname{Pr}\left(C_{i, l}\right) \exp \left(-\left(N_{i}-l\right) \frac{K_{n}(x+1)}{P_{n}}\right) .
\end{aligned}
$$


First, we will give an upper bound on $\operatorname{Pr}\left(U_{i, l} \leq x\right)$ because

$$
\begin{aligned}
& \operatorname{Pr}\left(U_{i, l} \leq x\right)=\operatorname{Pr}\left(U_{i, l} \leq(1-\epsilon) l K_{n}\right) \\
& \quad \leq\left(\begin{array}{c}
P_{n} \\
(1-\epsilon) l K_{n}
\end{array}\right)\left(\frac{(1-\epsilon) l K_{n}}{P_{n}}\right)^{l K_{n}} \\
& \quad \leq\left(\frac{e P_{n}}{(1-\epsilon) l K_{n}}\right)^{(1-\epsilon) l K_{n}}\left(\frac{(1-\epsilon) l K_{n}}{P_{n}}\right)^{l K_{n}} \\
& \quad \leq e^{l K_{n}}\left(\frac{l K_{n}}{P_{n}}\right)^{\epsilon K_{n} l} .
\end{aligned}
$$

Since $P_{n} \geq n$ and $n \pi r_{n}^{2}=d_{n}$, where $d_{n}=\omega(\log n), d_{n}=$ $o\left(n^{(1 / 4)(1-\delta)}\right)$, we have

$$
\begin{aligned}
\frac{K_{n} l}{P_{n}} & =\sqrt{\frac{P_{n}\left(2 \log n+c_{n}\right)}{d_{n}}} \frac{l}{P_{n}} \leq \frac{l}{d_{n}} \sqrt{2 \log n} \\
& \leq R(\log n)^{-1 / 2} .
\end{aligned}
$$

Since $K_{n} \rightarrow \infty$ we have $\varepsilon K_{n} \rightarrow \infty$ and thus for sufficiently large $n$

$$
\begin{aligned}
\operatorname{Pr}\left(U_{i, l} \leq x\right) & =\operatorname{Pr}\left(U_{i, l} \leq(1-\epsilon) l K_{n}\right) \\
& \leq\left[\left(\frac{e^{1 / \varepsilon} K_{n} l}{P_{n}}\right)^{\varepsilon K_{n}}\right]^{l} \leq n^{-l} .
\end{aligned}
$$

Then we have

$$
\begin{aligned}
& \frac{1}{s_{n}^{2}} \sum_{L} P\left(U_{i, l} \leq x\right) \exp \left(-\left(N_{i}-l\right) \frac{K_{n}^{2}}{P_{n}}\right) \\
& \quad \leq \frac{1}{s_{n}^{2}} \sum_{l=2}^{R}\left(\begin{array}{c}
N_{i} \\
l
\end{array}\right) P\left(U_{i, l} \leq x\right) \exp \left(-\left(N_{i}-l\right) \frac{K_{n}^{2}}{P_{n}}\right) \\
& \quad \leq \frac{1}{s_{n}^{2}} \sum_{l=2}^{R}\left(\begin{array}{c}
N_{i} \\
l
\end{array}\right) n^{-l} n^{-\left(1-\delta_{1}\right) / 2+2 R / d_{n}} \\
& \quad \leq \frac{1}{s_{n}^{2}} n^{-\left(1-\delta_{1}\right) / 2+2 R / d_{n}}\left(N_{i}^{2} n^{-2}+\cdots+N_{i}^{R} n^{-R}\right) \\
& \quad \leq \frac{1}{s_{n}^{2}} n^{-\left(1-\delta_{1}\right) / 2+2 R / d_{n}}\left(\frac{1+\delta_{1}}{2} n \pi s_{n}^{2}\right)^{2} n^{-2}(1+o(1)) \\
& \quad \leq \frac{\pi\left(1+\delta_{1}\right)^{2}}{8} d_{n} n^{-\left(1-\delta_{1}\right) / 2+2 R / d_{n}-1} \longrightarrow 0 .
\end{aligned}
$$

The second inequality is true since $N_{i} \geq\left(\left(1-\delta_{1}\right) / 2\right) n \pi s_{n}^{2}$ and $2 n \pi s_{n}^{2}=d_{n}$. The last step holds since $d_{n}$ satisfies $d_{n}=\omega(\log n)$ and $d_{n}=o\left(n^{(1 / 4)(1-\delta)}\right)$.
The following term satisfies

$$
\begin{aligned}
& \frac{1}{s_{n}^{2}} \sum_{L} \operatorname{Pr}\left(C_{i, l}\right) \exp \left(-\left(N_{i}-l\right) \frac{K_{n}(x+1)}{P_{n}}\right) \\
& =\frac{1}{s_{n}^{2}} \sum_{l=2}^{R}\left(\begin{array}{c}
N_{i} \\
l
\end{array}\right) \operatorname{Pr}\left(C_{i, l}\right) \exp \left(-\left(N_{i}-l\right) \frac{K_{n}(x+1)}{P_{n}}\right) .
\end{aligned}
$$

Because $\left|N_{i}-(\pi / 2) n s_{n}^{2}\right| \leq \delta_{1}(\pi / 2) n s_{n}^{2}$, we also have

$$
\begin{aligned}
& \frac{1}{s_{n}^{2}} \sum_{L} \operatorname{Pr}\left(C_{i, l}\right) \exp \left(-\left(N_{i}-l\right) \frac{K_{n}(x+1)}{P_{n}}\right) \leq \frac{1}{s_{n}^{2}} \\
& \cdot \sum_{l=2}^{R}\left(\frac{e N_{i}}{l}\right)^{l} l^{l-2}\left(\frac{K_{n}^{2}}{P_{n}}\right)^{l-1} \\
& \cdot \exp \left(-(1-\epsilon) \frac{l\left(N_{i}-l\right)}{N_{i}} \frac{K_{n}^{2} N_{i}}{P_{n}}\right) \leq \frac{1}{s_{n}^{2}} \\
& \cdot \sum_{l=2}^{R} \frac{\left(e\left(1+\delta_{1}\right) / 2\right)^{l}}{l^{2}} n \pi s_{n}^{2}\left(n \pi s_{n}^{2} \frac{K_{n}^{2}}{P_{n}}\right)^{l-1} \\
& \cdot \exp \left(-(1-\epsilon) \frac{N_{i}-l}{N_{i}} \frac{l K_{n}^{2}}{P_{n}}\left(1-\delta_{1}\right) \frac{n \pi s_{n}^{2}}{2}\right) \\
& \quad \leq \sum_{l=2}^{R} \frac{\left(e\left(1+\delta_{1}\right) / 2\right)^{l}}{l^{2}} n \pi\left(\frac{2 \log n+c_{n}}{2}\right)^{l-1} \\
& \cdot \exp \left(-\frac{\left(1-\delta_{1}\right)(1-\epsilon)}{2} \frac{l\left(N_{i}-l\right)}{N_{i}} \frac{2 \log n+c_{n}}{2}\right) \\
& \quad \leq \sum_{l=2}^{R} \frac{\left(e\left(1+\delta_{1}\right) / 2\right)^{l}}{l^{2}} \pi \\
& \cdot \exp \left((l-1) \log \left(\frac{2 \log n+c_{n}}{2}\right)\right. \\
& \left.\quad l \frac{\left(1-\delta_{1}\right)(1+\epsilon)}{2} \log n+\log n\right) .
\end{aligned}
$$

Since $\delta_{1} /\left(1-\delta_{1}\right)<\epsilon$, we get $\left(1-\delta_{1}\right)(1+\epsilon)>1$. Then for sufficiently large $n$, from above discussion, we have

$$
\begin{aligned}
& \frac{1}{s_{n}^{2}} \sum_{l=2}^{R}\left(\begin{array}{c}
N_{i} \\
l
\end{array}\right) \operatorname{Pr}\left(C_{i, l}\right) \exp \left(-\left(N_{i}-l\right) \frac{K_{n}(x+1)}{P_{n}}\right) \\
& \quad \longrightarrow 0
\end{aligned}
$$

Hence the probability that the subgraph $G_{i}$ has a component of size $l, 2 \leq l \leq R$ is

$$
\frac{1}{s_{n}^{2}} \sum_{l=2}^{R}\left(\begin{array}{c}
N_{i} \\
l
\end{array}\right) \operatorname{Pr}\left(A_{i, l}\right) \longrightarrow 0
$$


Case $2\left(R+1 \leq l \leq L_{1}(n)\right)$. Here $L_{1}(n)=\min \left\{P_{n} / K_{n}, N_{i} / 2\right\}$. In this case, we assume that the component $L$ is assigned at most $(1 / 4) K_{n} l$ keys. The probability that the subgraph $G_{i}$ contains a component of size $l, R+1 \leq l \leq \min \left\{P_{n} / K_{n}, N_{i} / 2\right\}$, is

$$
\begin{aligned}
& \sum_{L} \operatorname{Pr}\left(A_{i, l}\right) \\
& \quad \leq \sum_{L} \operatorname{Pr}\left(U_{i, l} \leq x\right) \\
& \quad+\sum_{L} \operatorname{Pr}\left(C_{i, l}\right) \exp \left(-\left(N_{i}-l\right) \frac{K_{n}(x+1)}{P_{n}}\right) .
\end{aligned}
$$

The above bound is true since the expression $\exp \left(-\left(N_{i}-\right.\right.$ l) $\left.K_{n}^{2} / P_{n}\right) \leq 1$, where we are summing over all subsets of nodes of size $l, R+1 \leq l \leq \min \left\{P_{n} / K_{n}, N_{i} / 2\right\}$. We wish to prove that this sum tends to 0 as $n \rightarrow \infty$.

Now we prove that $\sum_{L} \operatorname{Pr}\left(U_{i, l} \leq x\right) \rightarrow 0$ as $n \rightarrow \infty$. Similar to the argument in the proof of case 1 , we get

$$
\begin{aligned}
\sum_{L} \operatorname{Pr}\left(U_{i, l} \leq x\right) & \leq \sum_{l=R+1}^{L_{1}(n)}\left(\begin{array}{c}
N_{i} \\
l
\end{array}\right)\left(\begin{array}{c}
P_{n} \\
\frac{1}{4} l K_{n}
\end{array}\right)\left(\frac{l K_{n}}{4 P_{n}}\right)^{l K_{n}} \\
& \leq \sum_{l=R+1}^{L_{1}(n)}\left(\begin{array}{c}
P_{n} \\
l
\end{array}\right)\left(\begin{array}{c}
P_{n} \\
\frac{1}{4} l K_{n}
\end{array}\right)\left(\frac{l K_{n}}{4 P_{n}}\right)^{l K_{n}} \\
& \leq \sum_{l=R+1}^{L_{1}(n)}\left(\begin{array}{c}
P_{n} \\
\frac{1}{4} l K_{n}
\end{array}\right)^{2}\left(\frac{l K_{n}}{4 P_{n}}\right)^{l K_{n}} \\
& \leq \sum_{l=1}^{\min \left\{P_{n} / K_{n}, N_{i} / 2\right\}}\left(\frac{e K_{n} l}{4 P_{n}}\right)^{K_{n} l / 2} .
\end{aligned}
$$

The summand in this last expression may be written in the form $\left(y^{y}\right)^{t}$, where $y=e K_{n} l / 4 P_{n}$ and $t=2 P_{n} / e$. Since $y^{y}$ has no internal maximal value, our summand is maximized at the extremes of its range. Let $\mu$ denote the maximum of the summand; here

$$
\begin{aligned}
\mu & =\max \left\{\left(\frac{e K_{n}}{4 P_{n}}\right)^{K_{n} / 2},\left(\frac{e}{4}\right)^{P_{n} / 2},\left(\frac{e}{4}\right)^{N_{i} K_{n} / 4}\right\} \\
& =o\left(n^{-1}\right) .
\end{aligned}
$$

As $K_{n} \rightarrow \infty$, we have

$$
\begin{gathered}
\frac{1}{s_{n}^{2}} \sum_{l=R+1}^{L_{1}(n)}\left(\begin{array}{c}
N_{i} \\
l
\end{array}\right) \operatorname{Pr}\left(U_{i, l} \leq x\right) \leq \frac{1}{s_{n}^{2}}\left(\frac{N_{i}}{2}+1\right) \mu \\
\quad \leq \frac{1}{s_{n}^{2}} \frac{1+\delta_{1}}{2} n \pi s_{n}^{2} \mu \leq \frac{1+\delta_{1}}{2} n \pi \mu=o(1) .
\end{gathered}
$$

Now consider the second term in (45)

$$
\begin{gathered}
\frac{1}{s_{n}^{2}} \sum_{l=R+1}^{L_{1}(n)}\left(\begin{array}{c}
N_{i} \\
l
\end{array}\right) \operatorname{Pr}\left(C_{i, l}\right) e^{-\left(N_{i}-l\right) K_{n}(x+1) / P_{n}} \leq \frac{1}{s_{n}^{2}} \\
\cdot \sum_{l=R+1}^{L_{1}(n)}\left(\begin{array}{c}
N_{i} \\
l
\end{array}\right) l^{l-2} \beta_{n}^{l-1} \exp \left(-\left(N_{i}-l\right) \frac{l K_{n}^{2}}{4 P_{n}}\right) \leq \frac{1}{s_{n}^{2}} \\
\cdot \sum_{l=R+1}^{L_{1}(n)} \frac{\left(\left(1+\delta_{1}\right) e\right)^{l}}{l^{2}}\left(\frac{\pi}{2} n s_{n}^{2}\right)\left(\frac{\pi}{2} n s_{n}^{2} \frac{K_{n}^{2}}{P_{n}}\right)^{l-1} \\
\cdot \exp \left(-\left(N_{i}-l\right) \frac{l K_{n}^{2}}{4 P_{n}}\right) \\
\quad \leq \sum_{l=R+1}^{L_{1}(n)} \frac{\left(\left(1+\delta_{1}\right) e\right)^{l}}{l^{2}} \frac{\pi n}{2}\left(\frac{2 \log n+c_{n}}{4}\right)^{l-1} \\
\cdot \exp \left(-\left(1-\delta_{1}-\frac{2 l}{\pi n s_{n}^{2}}\right) \frac{(l-1) \log n}{8}\right) .
\end{gathered}
$$

Rewrite the above using the notation

$$
a_{n}(l)=\frac{\left(1+\delta_{1}\right) e\left(2 \log n+c_{n}\right)}{4 \exp \left(\left(1-\delta_{1}-2 l / \pi n s_{n}^{2}\right) \log n / 8\right)},
$$

and then

$$
\begin{aligned}
& \sum_{l=R+1}^{L_{1}(n)} \frac{\left(\left(1+\delta_{1}\right) e\right)^{l}}{l^{2}} \frac{\pi n}{2}\left(\frac{2 \log n+c_{n}}{4}\right)^{l-1} \\
& \cdot \exp \left(-\left(1-\delta_{1}-\frac{2 l}{\pi n s_{n}^{2}}\right) \frac{(l-1) \log n}{8}\right) \leq \frac{\pi n}{2} \\
& \cdot \sum_{l>R} a_{n}(l)^{l-1} \leq \frac{\pi n}{2} \frac{a_{n}(l)^{R}}{1-a_{n}(l)} .
\end{aligned}
$$

The last step is true since $1-\delta_{1}-2 l / \pi n s_{n}^{2}>0$, so for some appropriate $a>0$,

$$
\begin{aligned}
& \frac{1}{s_{n}^{2}} \sum_{l=R+1}^{L_{1}(n)}\left(\begin{array}{c}
N_{i} \\
l
\end{array}\right) \operatorname{Pr}\left(C_{i, l}\right) \exp \left(-\left(N_{i}-l\right) \frac{K_{n}(x+1)}{P_{n}}\right) \\
& \quad \leq \frac{1}{n^{a}} .
\end{aligned}
$$

Case $3\left(L_{1}(n)<l \leq N_{i} / 2: L_{1}(n)=\min \left\{P_{n} / K_{n}, N_{i} / 2\right\}\right)$. In this case, the component $L$ of circle cell $i$ is assigned at most $P_{n} / 4$ keys; we have that

$$
\begin{aligned}
\sum_{L} \operatorname{Pr}\left(A_{i, l}\right) & \\
\leq & \sum_{L} \operatorname{Pr}\left(U_{i, l} \leq x\right) \\
& +\sum_{L} \operatorname{Pr}\left(C_{i, l}\right) \exp \left(-\left(N_{i}-l\right) \frac{K_{n}(x+1)}{P_{n}}\right) .
\end{aligned}
$$


Now we first show $\left(1 / s_{n}^{2}\right) \sum_{L} \operatorname{Pr}\left(U_{i, l} \leq x\right) \rightarrow 0$ as $n \rightarrow \infty$ :

$$
\begin{aligned}
\frac{1}{s_{n}^{2}} \sum_{L} \operatorname{Pr}\left(U_{i, l} \leq x\right) \leq \frac{1}{s_{n}^{2}} \sum_{l=L_{1}(n)}^{N_{i} / 2}\left(\begin{array}{c}
N_{i} \\
l
\end{array}\right)\left(\begin{array}{c}
P_{n} \\
\frac{1}{4} P_{n}
\end{array}\right)\left(\frac{1}{4}\right)^{l K_{n}} \\
\quad \leq \frac{1}{s_{n}^{2}} \sum_{l=L_{1}(n)}^{N_{i} / 2}\left(\begin{array}{c}
N_{i} \\
l
\end{array}\right)(4 e)^{P_{n} / 4} 4^{-P_{n}} \leq \frac{1}{s_{n}^{2}} 2^{N_{i}} \exp \left(\frac{P_{n}}{4}\right. \\
\left.-\frac{3}{2} P_{n} \log 2\right) \leq \exp \left(\frac{1+\delta_{1}}{4} d_{n} \log 2-\frac{3}{2} P_{n} \log 2\right. \\
\left.+\frac{P_{n}}{4}-\log \left(\frac{d_{n}}{2 \pi}\right)+\log n\right) \\
\quad \leq \exp \left(-P_{n}\left(\left(\frac{3}{2} \log 2-\frac{1}{4}\right)+\frac{1+\delta_{1}}{4} \frac{d_{n}}{P_{n}} \log 2\right.\right. \\
\left.\left.\quad-\frac{\log \left(d_{n} / 2 \pi\right)}{P_{n}}+\frac{\log n}{P_{n}}\right)\right) .
\end{aligned}
$$

The third inequality uses $\sum_{l=L_{1}(n)}^{N_{i} / 2}\left(\begin{array}{c}N_{i} \\ l\end{array}\right) \leq 2^{N_{i}}$. Since $P_{n}>$ $n, d_{n}=o\left(n^{(1 / 4)(1-\delta)}\right)$, the final expression tends to 0 .

The term $\left(1 / s_{n}^{2}\right) \sum_{L} \operatorname{Pr}\left(C_{i, l}\right) \exp \left(-\left(N_{i}-l\right) K_{n}(x+1) / P_{n}\right)$ is bounded as follows.

$$
\begin{aligned}
& \frac{1}{s_{n}^{2}} \sum_{l=L_{1}(n)}^{N_{i} / 2}\left(\begin{array}{c}
N_{i} \\
l
\end{array}\right) \operatorname{Pr}\left(C_{i, l}\right) \exp \left(-\left(N_{i}-l\right) \frac{K_{n}(x+1)}{P_{n}}\right) \\
& \quad \leq \frac{1}{s_{n}^{2}} \sum_{l=L_{1}(n)}^{N_{i} / 2}\left(\begin{array}{c}
N_{i} \\
l
\end{array}\right) \exp \left(-\frac{K_{n} N_{i}}{8}\right) \leq \exp \left(-\frac{1}{8} N_{i} K_{n}\right. \\
& \left.\quad+N_{i} \log 2-\log s_{n}^{2}\right) \leq \exp \left(-\frac{1-\delta_{1}}{2} n \pi s_{n}^{2}\left(\frac{K_{n}}{8}\right.\right. \\
& \left.\quad-\log 2)-\log \left(\frac{d_{n}}{2 \pi}\right)+\log n\right) \leq \exp \left(-\frac{d_{n}}{4}(1\right. \\
& \left.\left.\quad-\delta_{1}\right)\left(\frac{K_{n}}{8}-\log 2\right)-\log \left(\frac{d_{n}}{2 \pi}\right)+\log n\right) \\
& \quad \leq \exp \left(-d_{n}\left(\frac{1-\delta_{1}}{4}\left(\frac{K_{n}}{8}-\log 2\right)-\frac{\log \left(d_{n} / 2 \pi\right)}{d_{n}}\right.\right. \\
& \left.\left.\quad+\frac{\log n}{d_{n}}\right)\right) \cdot
\end{aligned}
$$

The first inequality uses $\operatorname{Pr}\left(C_{i, l}\right) \leq 1$ and $l<N_{i} / 2$. Since $d_{n}=$ $\omega(\log n)$ and $d_{n}=o\left(n^{(1 / 4)(1-\delta)}\right)$, then for any $K_{n}>8 \log 2$, the last expression tends to 0 .

Therefore the probability that the subgraph $G_{i}$ has a component of size $l, L_{1}(n)<l \leq N_{i} / 2, L_{1}(n)=$ $\min \left\{P_{n} / K_{n}, N_{i} / 2\right\}$ is

$$
\frac{1}{s_{n}^{2}} \sum_{l=L_{1}(n)}^{N_{i} / 2}\left(\begin{array}{c}
N_{i} \\
l
\end{array}\right) \operatorname{Pr}\left(A_{i, l}\right) \longrightarrow 0 .
$$

From the above discussions, we have shown that for every circle cell $i, 1 \leq i \leq 1 / s_{n}^{2}$, the probability for the existence of component of size $l, 1 \leq l \leq N_{i} / 2$, tends to 0 as $n$ tends to infinity, which concludes Lemma 4.

We have proved that the overlapping structure of two adjacent circle cells contains at least one node and every circle cell is connected. The above two conditions ensure that $G\left(P_{n}, K_{n}, r_{n}\right)$ is connected. Combining Lemma 3 with Lemma 4 , the statement (ii) of Theorem 1 is established.

\section{Conclusion and Future Work}

Connectivity in $G\left(P_{n}, K_{n}, r_{n}\right)$ is the core subject of our paper. We obtain the zero-one law for graph connectivity in $G\left(P_{n}, K_{n}, r_{n}\right)$ under the given conditions. In order to get zeroone law for graph connectivity, we initially use the second moment method to get the 0-statement; then we show that any two adjacent circle cells share at least one common node and prove that any circle cell is connected to get the 1statement.

We conjecture that it is possible to prove a sharper connectivity threshold for random graph $G\left(P_{n}, K_{n}, r_{n}\right)$. Indeed, we believe that the following conjecture is true.

Conjecture 5. Let $K_{n} \geq 2, K_{n}, P_{n} \rightarrow \infty, K_{n}^{2} / P_{n} \rightarrow 0, P_{n} \geq$ $n$, and

$$
\pi r_{n}^{2} \frac{K_{n}^{2}}{P_{n}}=\frac{\log n+c_{n}}{n} .
$$

(i) If $c_{n} \rightarrow-\infty$, then $\operatorname{whp} G\left(P_{n}, K_{n}, r_{n}\right)$ is disconnected.

(ii) If $c_{n} \rightarrow c$, then the probability that $G\left(P_{n}, K_{n}, r_{n}\right)$ is connected tends to $e^{-e^{-c}}$.

(iii) If $c_{n} \rightarrow \infty$, then $w h p G\left(P_{n}, K_{n}, r_{n}\right)$ is connected.

The results in this paper hold under the condition that $d_{n}=\omega(\log n)$ and $d_{n}=o\left(n^{(1-\delta) / 4}\right)$. It would be interesting to see whether such results could be established under the condition that $d_{n}=\omega(\log n)$ and $d_{n}=o(n)$, which remains an open research challenge.

\section{Conflict of Interests}

The authors declare that there is no conflict of interests regarding the publication of this paper.

\section{Acknowledgments}

The work was supported by National Natural Science Foundation of China (NSFC) under Grant no. 11071272 and the project sponsored by the scientific research foundation for the returned overseas scholars of the Education Ministry of China.

\section{References}

[1] L. Eschenauer and V. D. Gligor, "A key-management scheme for distributed sensor networks," in Proceedings of the 9th ACM Conference on Computer and Communications Security, pp. 4147, ACM, November 2002. 
[2] I. F. Akyildiz, T. Melodia, and K. R. Chowdury, "Wireless multimedia sensor networks: a survey," IEEE Wireless Communications, vol. 14, no. 6, pp. 32-39, 2007.

[3] S. R. Blackburn and S. Gerke, "Connectivity of the uniform random intersection graph," Discrete Mathematics, vol. 309, no. 16, pp. 5130-5140, 2009.

[4] E. Godehardt, J. Jaworski, and K. Rybarczyk, "Random intersection graphs and classification," in Advances in Data Analysis, Studies in Classification, Data Analysis, and Knowledge Organization, pp. 67-74, Springer, Berlin, Germany, 2007.

[5] O. Yagăn and A. M. Makowski, "Zero-one laws for connectivity in random key graphs," IEEE Transactions on Information Theory, vol. 58, no. 5, pp. 2983-2999, 2012.

[6] K. Rybarczyk, "Diameter, connectivity, and phase transition of the uniform random intersection graph," Discrete Mathematics, vol. 311, no. 17, pp. 1998-2019, 2011.

[7] M. D. Penrose, "On $k$-connectivity for a geometric random graph," Random Structure and Algorithms, vol. 15, no. 2, pp. 145164, 1999.

[8] G. Mao and B. D. O. Anderson, "Towards a better understanding of large-scale network models," IEEE/ACM Transactions on Networking, vol. 20, no. 2, pp. 408-421, 2012.

[9] C.-W. Yi, P.-J. Wan, X.-Y. Li, and O. Frieder, "Asymptotic distribution of the number of isolated nodes in wireless ad hoc networks with bernoulli nodes," IEEE Transactions on Communications, vol. 54, no. 3, pp. 510-517, 2006.

[10] O. Yagăn, "Performance of the Eschenauer-Gligor key distribution scheme under an ON/OFF channel," IEEE Transactions on Information Theory, vol. 58, no. 6, pp. 3821-3835, 2012.

[11] R. D. Pietro, L. V. Mancini, A. Mei, A. Panconesi, and J. Radhakrishnan, "Connectivity properties of secure wireless sensor networks," in Proceedings of the 2nd ACM Workshop on Security of Ad Hoc and Sensor Networks Association for Computing Machinery (SASN '04), pp. 53-58, Washington, DC, USA, October 2004.

[12] K. Krzywdziński and K. Rybarczyk, "Geometric graphs with randomly deleted edges-connectivity and routing protocols," in Mathematical Foundations of Computer Science 2011, pp. 544555, Springer, Berlin, Germany, 2011.

[13] B. S. Krishnan, A. Ganesh, and D. Manjunath, "On connectivity thresholds in superposition of random key graphs on random geometric graphs," in Proceedings of the IEEE International Symposium on Information Theory Proceedings (ISIT '13), pp. 2389-2393, IEEE, Istanbul, Turkey, July 2013.

[14] S. Janson, T. Łuczak, and A. Ruciński, Random Graphs, Wiley, New York, NY, USA, 2000.

[15] M. D. Penrose, Random Geometric Graphs, Oxford University Press, Oxford, UK, 2003.

[16] J. Zhao, O. Yagăn, and V. Gligor, "Connectivity in secure wireless sensor networks under transmission constraints," in Proceedings of the 52nd Annual Allerton Conference on Communication, Control, and Computing (Allerton), pp. 1294-1301, Monticello, Va, USA, September 2014. 


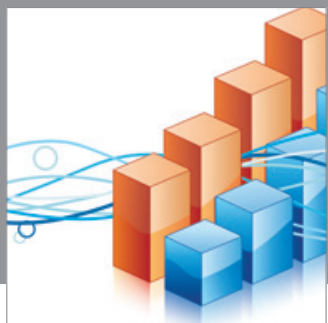

Advances in

Operations Research

mansans

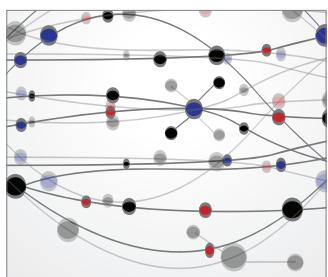

The Scientific World Journal
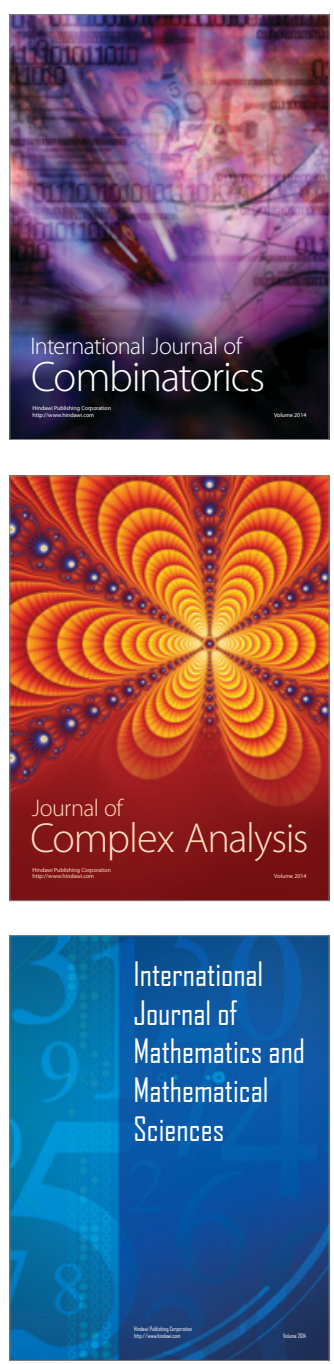
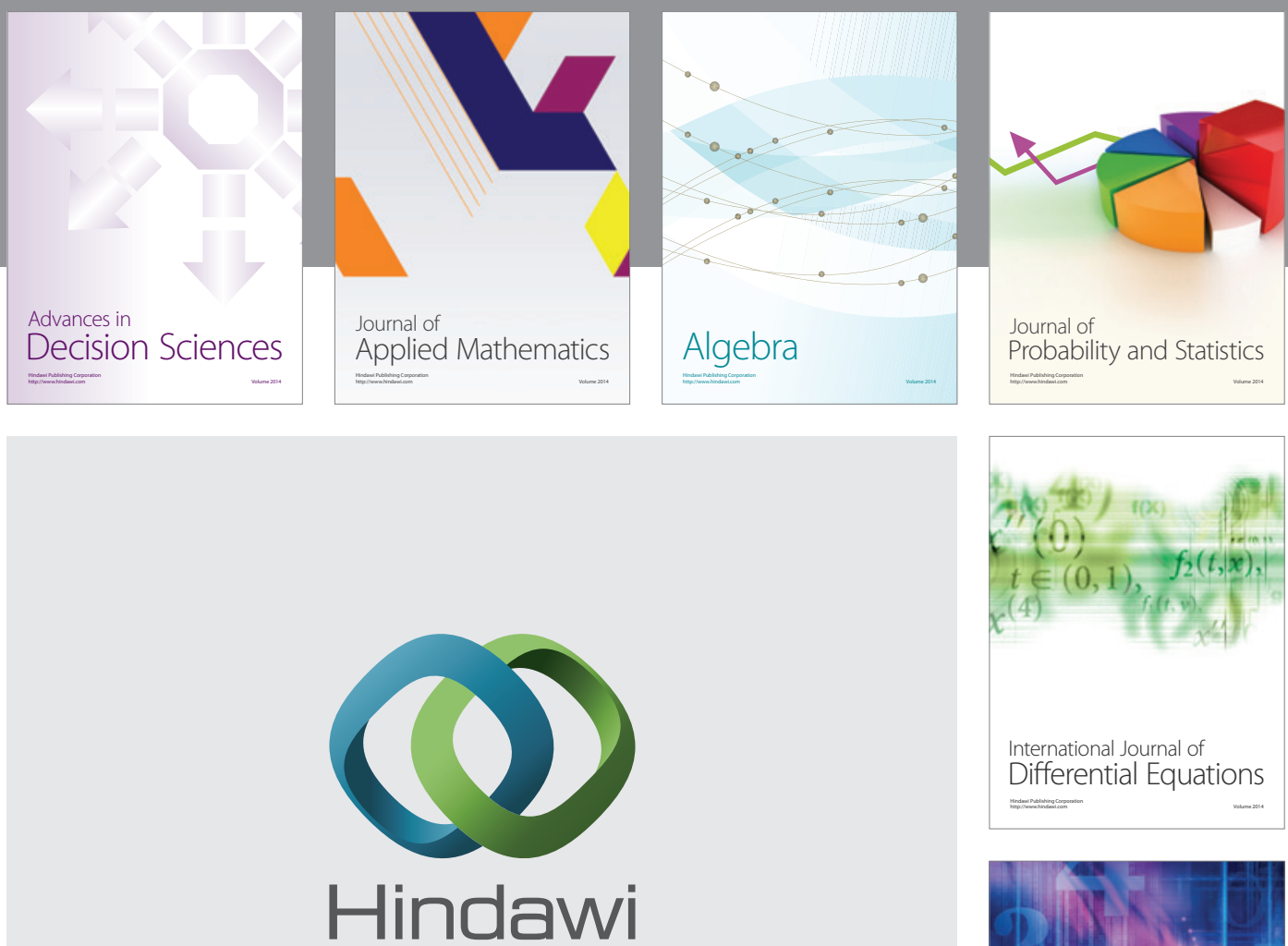

Submit your manuscripts at http://www.hindawi.com
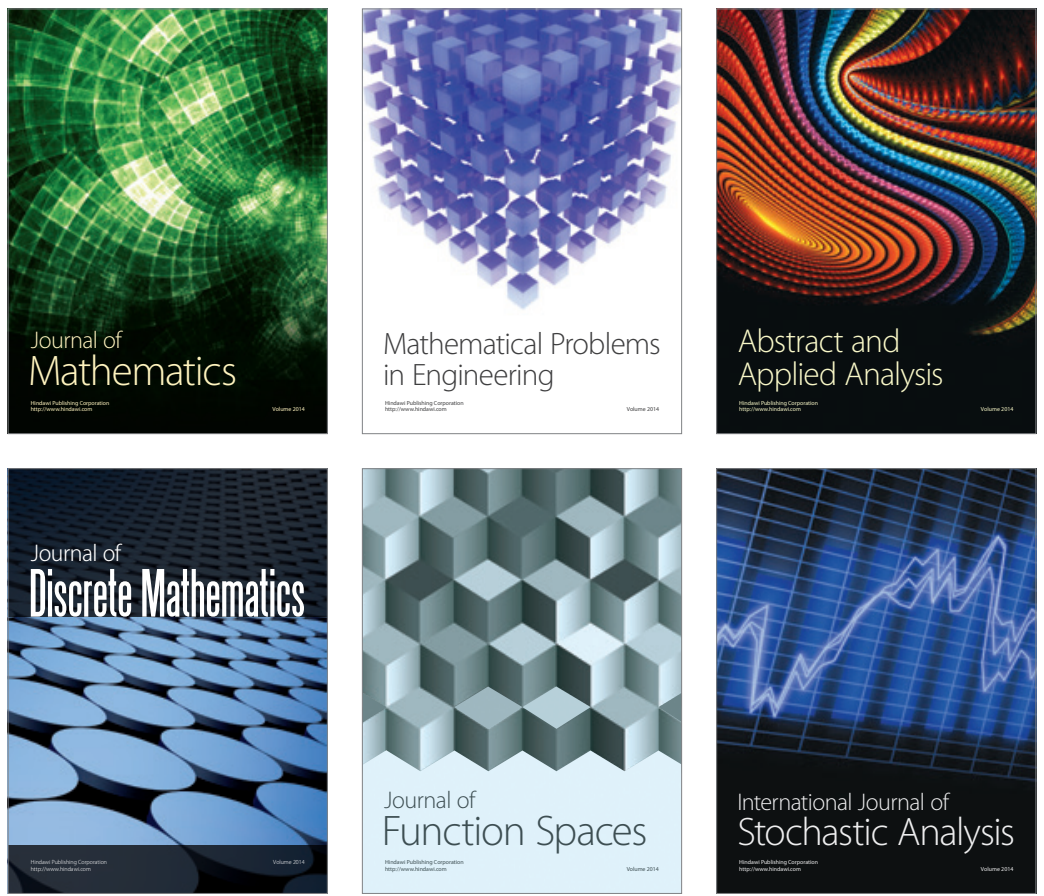

Journal of

Function Spaces

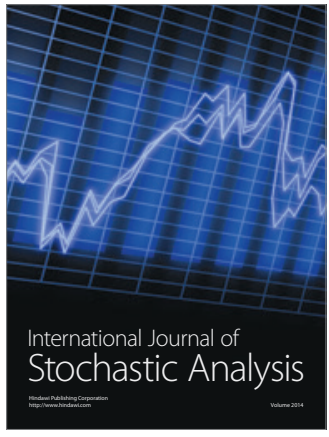

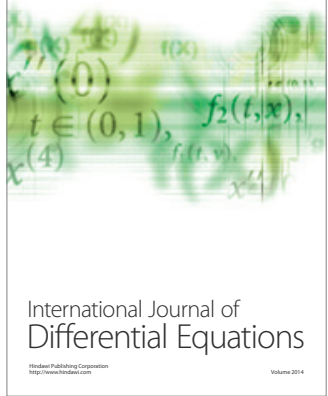
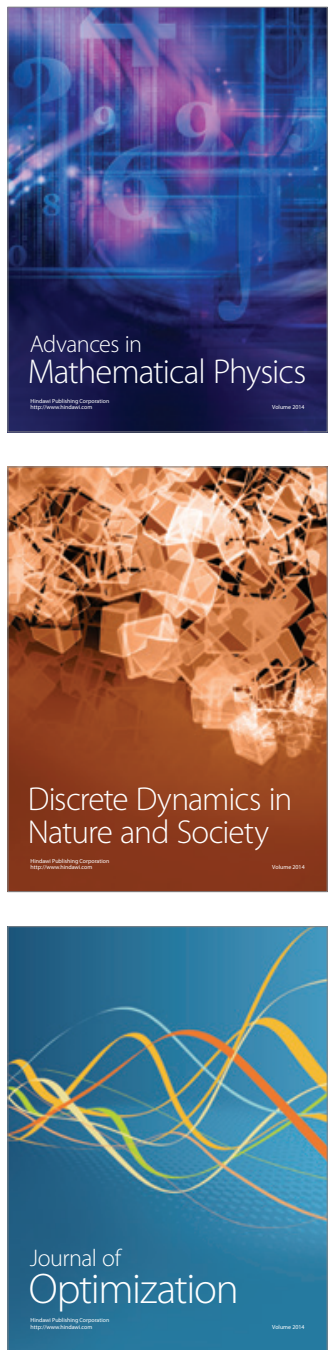